\title{
A Comparative Analysis of Nosocomial Infections between Internal and Surgical Intensive Care Units of a University Hospital in Birjand, Iran from 2015 to 2016: A Retrospective Study
}

\author{
Azadeh Ebrahimzadeh \\ Birjand University of Medical Sciences \\ Elaheh Allahyari \\ Birjand University of Medical Sciences \\ Fatemeh Nikoomanesh \\ Birjand University of Medical Sciences https://orcid.org/0000-0002-2097-7973 \\ Majid Zare_bidaki ( $\square$ m.zare@live.co.uk) \\ Birjand University of Medical Sciences
}

Research article

Keywords: Nosocomial Infections, Risk factors, Intensive care unit, Surgical, Internal

Posted Date: August 10th, 2020

DOl: https://doi.org/10.21203/rs.3.rs-42477/v1

License: (c) (i) This work is licensed under a Creative Commons Attribution 4.0 International License.

Read Full License 


\section{Abstract}

Background:

The aim of this study was to investigate and then to compare the prevalence of nosocomial infections (NIs) in the patients admitted to the surgery and internal Intensive Care Units(ICU) as well as studying the risk factors involved.

Methods:

In this cross-sectional descriptive, patients admitted to ICUs were studied over a year .Clinical data of patients, including demographic information, length of stay, underlining disease, the rate of patient with $\mathrm{Nls}$ and distribution of Nls sites and pathogens were collected. Univariate and multivariate logestic regression were carried out to determine the factors associated with NIs.

Results:

A total of 1018 patients were studiedincluding surgical ICU $(n=665)$ and internal ICU $(n=353)$. The incidence rate of $\mathrm{NI}$ in surgical and internal ICUs was $67(10.1 \%)$ and $96(27.2 \%)$, respectively. The most common $\mathrm{NI}$ in the internal ICU were respiratory tract infections( RTI ,46.9\%) and urinary tract infections (UTI, 37.5\%), while in the surgical ICU, the most common infections were respiratory tract infections (RTI, $38.3 \%$ ) and surgical-site infections (SSI, 22.0\%), respectively. The major factors the length of stay and the use of nasogastric intubation (NG tubes) were associated with NIs in both ICUs.

Conclusions:

The incidence of infections in the internal ICU were more than the surgical ICU. Age, underlying diseases, the long stay, used of ventilator and NG tube were factors associated with NIs rate in internal ICU.

Trial registration:

This study was the result of two research works with ethics code (IR.BUMS.REC.1394-17 and IR.BUMS.REC.1394-27).

\section{Background:}

Hospital-acquired infections are also called nosocomial infections (NIs), and can be defined as infections, which health care acquired infection [1]. Nls is considered as an important health problem issue worldwide, and also, this type of infection occurs in the patients under medical cares in hospitals. Based on the national nosocomial infection surveillance (NNIS) system, these infections can be classified into four major groups as follows: pulmonary, urinary tract, blood stream,and surgical site infections[1]. Most of the nosocomial infections'frequency (above 20\%) occur in intensive care units (ICU) [2]. In this regard, 
invasive devices such as catheters and ventilators employed in modern health care can cause these infections[3].

Today, NIs are among the major challenges in hospitals and medical centers, and are also considered as a significant cause of the increased mortality and morbidity rates. According to World Health Organization (WHO) investigation in 55 hospitals from 14 countries, it was indicated that, on average, $8.7 \%$ of the hospitalized patients had NI. Over 1.4 million people worldwide are suffering from the complications of nosocomial infection[4]. In previous studies, the highest prevalence of NI was reported in ICU, surgical, and orthopedics units [5]. Moreover, the most frequent kinds of infections include central line-associated blood system infections, catheter-associated urinary tract infections, surgical site infections, and ventilator-associated pneumonia[6].

Pathogens responsible for $\mathrm{NI}$ are some types of bacteria, viruses, and fungi, which are mainly opportunistic and show a high rate of resistance to the antimicrobial medicines[7]. In addition, these microorganisms remained from the previous patients in hospitals, can be colonized on medical devices such as intravenous and urinary catheters, mechanical ventilation tubes, and nasogastric tubes (NG tube) [8]. With increasing infections, there is an increase in the prolonged hospital stay, long term disability, the increased antimicrobial resistance, which all of them may lead to the increased chance of Nls occurrence [9]. Internal intensive care units (I-ICUs) and medical surgical intensive care units (MS-ICUs) are two medical units, which are usually considered as unites with the relatively high rate of NIs. Therefore, we aimed of the present study was to compare the rate of incidence $\mathrm{NI}$ and risk factors in the internal intensive care unit (ICU) and medical surgical intensive care unit (MS-ICU).

\section{Methods:}

\section{Study subjects}

This corss-sectional retrospective study was performed on patients admitted at medical surgical ICU (MSICU) and internal ICU of university hospital, Birjand, Iran from 2015to 2016. This study was the result of two research works with ethics code (IR.BUMS.REC.1394-17 and IR.BUMS.REC.1394-27). All patients with the initial positive culture as well as patients who had taken antibiotics for any reason in the past 72 hours were excluded from the study. Demographic data and clinical status (e.g. age, gender and length of stay) were recorded at admission to the ICU.

In this study, the samples included all blood, urine, sputum and wound were initially taken before antibiotic administration from patients hospitalized in the MS-ICU and internal ICU. In addition, the detailed information about types of medical devices used, such as urinary catheters and mechanical ventilation tube, central venous catheter, NG tube, and blood transfusion were recorded.

\section{Study Restrictions:}


A number of patients admitted to the ICU from 2015to 2016 were excluded in the study due to taking antibiotics. There were also cases where they appeared to be infected but did not have a positive culture and were excluded from the study.

\section{Infection identification}

All infections were diagnosed on the basis of clinical suspiciousness, radiological information and were confirmed by biochemical criteria, like positive cultures from different samples. Major infections, such as respiratory tract infections (RTIs), wound/ surgical-site infections (SSIs), blood stream infections (BSIs) and urinary tract infections (UTIS) diagnosed as follows:

RTIs included infections involving respiratory tract including bronchitis, pneumonitis and pneumonia; SSI included non-healing wound with or without discharge involving the sterna wound and/or leg or arm and culture positive from the discharges; BSI included positive pathogen-culturesofbloodsamples, each taken from one central and one peripheral site; UTI included more than $10^{5}$ colony forming unite (CFU) pathogen on culture.

All biological collected samples transferred to the hospital microbiological laboratory and were cultured in the lab according to the standard procedures for bacterial and fungal identification. After 48 hours, resampling was performed for patients who their samples initially showed negative prototype culture. In the next stage, all positive cultures were isolated and the microbial isolates were conducted to biomedical tests for identification the pathogens in a species level.

\section{Statistical analysis}

The collected data were statistically analysed by SPSS (V. 22) software using Chi-square and t-test. The normality of numeric variables was checked by Kolmogorov-Smirnov test. The association between NIs and potential risk factors was identified by Chi-square test in univariate analysis. Variables significantly different between two groups were analyzed in a forward stepwise logistic-regression model to confirm independent risk factors associated with Nls. Logestic regression analysis was performed to calculate the odds ratio (OR) and $95 \%$ confidence interval $(\mathrm{Cl})$ by using NIs as a dependent variable and the clinical data (ie. demographic information, used of medical devices) as independent variables. For all statistical analyses $P<0.050$ was considered significant.

\section{Results}

During this study period, 1018 patients were admitted into the ICU, 353 patients (mean age: $53.8 \pm$ 23.1years old) belonged to the internal ICU, and 665 patients (mean age: $46.1 \pm 26.0$ years old) belonged to the MS-ICU. The demographic and baseline ICU data of the patients are given in Tables 1.The most common causes of hospitalization in the ICUs were hypertension and chronic pulmonary heart disease,whereas the common underlying diseases in internal ICU were hyper tension and chronic lung disease with the frequency of $41.1 \%$ and $22.1 \%$, respectively. Also, in return hospitalized patients, the 
common underlying diseases in MS-ICU were hyper tension and heart disease with the frequency of $31.1 \%$ and $23.5 \%$, respectively. Moreover, a statistically significant correlation was observed between the groups(p-value $<0.001)$.

According to the positive culture of the patient's samples, the results have showed the prevalence of nosocomial infections in the internal ICU was as98 (27.2\%) from 353 patients, and in MS-ICU as 67 (10.1\%) from 665 patients. The most common nosocomial infections in the internal ICU are RTI (46.9\%) and UTI (37.5\%). While in the MS-ICU, the most common infection is RTI (38.3\%) followed by RTI \& SSI (22.0\%) (Figure1). The prevalence of nosocomial infections was significantly higher in the internal ICU compared to the MS-ICU ( $\mathrm{p}$-value $<0.001$ ).

The majority of microorganism was detected from the internal ICU samples including Pseudomonas aeruginosa (50\%) that was isolated from wound infections and pulmonary secretions $59.5 \%$ and Candida spp $(24.55 \%)$ that were detected from UTI. Whereas, in MS-ICU, Pseudomonas aeruginosa is the most frequently isolated pathogen from wound infection (17.5\%), E. coli from UTI (5.87\%), and Coagulasenegative Staphylococci (22.5\%) and Klebsiella (14.7\%) are the commonest causes of RTI (Figure 2).

The average length of hospital stay in internal ICU patients were $8.73 \pm 7.46$ days, and in MS-ICU were $8.11 \pm 4.8$ days, which was significantly higher among the internal ICU patients compared to the surgical ICU patients ( $p$-value=0.01). In both internal and MS-ICU, the prevalence of nosocomial infections significantly increased with the increase of age and hospital stay. Therefore, $11.6 \%$ of internal ICU and $11.7 \%$ of MS-ICU patients belonged to the age group of $71-80$ years old. On the other hand, $71.2 \%$ of internal ICU patients and $65.8 \%$ of surgical ICU patients with a hospital stay of more than 10 days had a positive culture infection. Notably is that, as the length of hospital stay in both groups increased, positive culture infection has also increased ( $p$-value $<0.001$, table2).

To identify the variables associated with NIs rates, a stepwise logistic regression analysis was performed separately in surgery and internal ICUs. Univariate analysis was performed with NIs status in ICUs as the dependent variable and potential Nls related indices (ie . infection, age, gender, length of stay, hypertension, chronic of heart disease, chronic of lung disease, chronic of renal disease, diabetes, cancer, blood transfusion, used of chest tube, NG tube, tracheal tube, central catheter) as the independent variable. The results of MS-ICU suggested that age, gender (female vs. male) underlining disease (no have Vs. have), blood transfusion (unused vs. used) and central venous catheter (unused vs. used), chest tube and tracheal tube (unused vs. used) were not related to NIs in ICU ( $p>0.05$, table 3). But infection (negative-culture vs. positive culture; $\mathrm{OR}=0.40 ; 95 \% \mathrm{Cl}=0.16-0.96 ; \mathrm{P}=0.045)$, the length of stay $(\mathrm{OR}=1.20$; $95 \% \mathrm{Cl}=1.05-1.38 ; \mathrm{P}=0.006)$, and $\mathrm{NG}$ tube $(\mathrm{OR}=0.86 ; 95 \% \mathrm{Cl}=0.74-1.00 ; \mathrm{P}=0.049)$ were factors associated with NIs in surgical ICU (table 3). The results of multivariate analysis also demonstrated that the only risk factors for NIs in MS-ICU are infection (negative-culture vs. positive culture; $\mathrm{OR}=0.40 ; 95 \% \mathrm{Cl}=0.16-0.96$; $P=0.04)$, the length of stay $(O R=0.83 ; 95 \% \mathrm{Cl}=0.73-0.75 ; P=0.006)$, and $N G$ tube $(O R=1.17 ; 95 \% \mathrm{Cl}=1.00-$ $1.36 ; \mathrm{P}=0.049$ ). 
The results of logestic regration analysis in internal ICU suggested that infection (negative-culture vs. positive culture), gender (female vs. male), hypertension (no have Vs. have), chronic of heart disease(no have Vs. have), chronic of renal disease (no have Vs. have), diabetes (no have Vs. have), blood transfusion(no have Vs. have), chest tube (unused vs. used) and central venous catheter (unused vs. used) were not related to NIs in internal ICU ( $p>0.05$, table 3$)$, but age (OR=0.96; $95 \% \mathrm{Cl}=0.95-0.98$; $\mathrm{P}<0.001)$, the length of stay $(\mathrm{OR}=0.67 ; 95 \% \mathrm{Cl}=1.38-2.02 ; \mathrm{P}<0.001)$, chronic of lung disease (no have vs. have; $\mathrm{OR}=0.34 ; 95 \% \mathrm{Cl}=0.16-0.72 ; \mathrm{P}=0.005$ ) 1 , cancer (no have vs. have; $\mathrm{OR}=0.04 ; 95 \% \mathrm{Cl}=0.00-0.32$; $\mathrm{P}=0.003)$, NG tube $(\mathrm{OR}=0.68 ; 95 \% \mathrm{Cl}=0.57-0.81 ; \mathrm{P}<0.001)$ and tracheal tube $(\mathrm{OR}=0.84 ; 95 \% \mathrm{Cl}=0.76-0.92$; $\mathrm{P}<0.001$ ) were factors associated with NIs in internal ICU (table 4). The results of multivariate analysis also demonstrated the same variable as risk factors for NIs in internal ICU.

\section{Discussion}

Despite progress in modern medical techniques and clinical application of various surgical operations, nosocomial infections (NIs) is considered as a major risk factor for the prognosis of patients [1].It is also notable that, Nls might not only be, directly or indirectly, the critical cause the death of patients, but also directly affect the treatment or operation of the patients and prolong their hospitalization. The primary objective of present study was investigate the rate of nosocomial infections (NIs) in two type of ICU (internal and medical surgery), and analysis the related risk factors Nls we observed that, infection (positive culture) incidence between internal ICU and MS-ICU was 27.2\% and 10.2\% from 2015 to 2016, respectively. Previous studies have demonstrated that, the suffering of infection in the internal ICU was greater than the MS-ICU, which was significant [7, 9, 10-12].

In addition, we observed that, a variety of risk factors may be associated with the occurrence of $\mathrm{NI}$ such as the length of stay and use of medical devices (NG tube and ventilator). Therefore, we should pay more attention to the affecting factors of the patients in ICU, who are known as the group with the high risk of $\mathrm{NI}$. In our study, we demonstrated that, the mean of length stay of internal ICU and MS-ICU was $8.73 \pm$ 7.46 and $8.11 \pm 4.8$ days, respectively. There was a significant relationship between the length of stay two ICUs and infection ( $p$-value $<0.001$ ).Also, there was no significant difference between the two groups in terms of age and gender; however, a relationship between age and the rate of infection was significant ( $p$ value $<0.001$ ). Ott et al. have revealed that, length of ICU stay was as one factor in occurrence of infection in internal ICU [13]. Increasing the length of stay in the ICU, both spontaneously and through the longer usage of ventilator and NG tube, can be considered as a factor in increasing internal ICU infections compared to surgery.

Moreover, in our study and other studies $[10,11,13,14-16]$, there was a direct relationship among the age, length of ICU stay, duration of catheterization, and infection, so that in both ICUs, this relationship was significant(p-value $<0.001)$, if the most common type of infection were UTI and RTI in each of ICUs. The presence of underlying diseases in internal ICU patients can be one of the causes of more infections in this group. In our study, in internal ICU, hypertension (41.1\%) was found to be the most common underlying disease followed by the chronic lung disease (22.1\%), Diabetes (16.7\%), and heart diseases 
(16.4\%). While hypertension (31.1\%) was the most common underlying disease followed by heart disease (23.5\%), chronic lung disease (21.1\%), and cancer (17.0\%) at surgical ICU (Table1). In this regard, underlying disease can cause people become more susceptible to further infection, as well as to the dangerous non-common pathogens, due to cellular immune deficiency and the increased susceptibility to infection, along with the need for long-term hospitalization for sugar control and also the need for broadspectrum antibiotics [17].

In our study, we found that, the most common infections in internal ICU were RTI and UTI $46.9 \%$ and $37.5 \%$, respectively. On the other hand, the most common infections in MS-ICU were RTI (38.3\%) and RTI\&SSI (22.0\%), respectively. High prevalence of RTI and pneumonia in both ICUs in our study and other studies is due to the long-term patient's intubation. All of the patients $(100 \%)$ have mechanical ventilation, and in addition to the infection caused by intubation; aspiration pneumonia and nosocomial pneumonia can also be named. In a preview study, The most common infection in both ICUs was pneumonia followed by UTI and septicemia and SSI, respectively $[11,18,19]$. In this regard, we demonstrated that Candida spp. was the most frequent isolated pathogens for UTI, and Pseudomonas aeruginosa was the most common pathogens for RTI, BSI, and SSI. In MS-ICU E.coli was the most isolated pathogens for UTI, Pseudomonas aeruginosa was the most common pathogens for RTI, and Staphylococci and Klebsiella spp. were responsible for most of the BSI and SSI (Fig. 2). According of other studies Pseudomonas aeruginosa was the most common pathogens that isolates from variety samples in ICU patients, followed by Staphylococci, Klebsiella, E.coli were as the common agents of infection [7, 20, 2].

Notably, the incidence of $\mathrm{NI}$ in internal ICU (27.2\%) was significantly more than MS-ICU (10.2\%). In this regard, we should pay more attention to the influencing factors of critical patients who admitted in internal ICU. In our study, we found that rate of infection, length of ICU stay and used of NG tube were related with NIs rate in surgical ICU. However, age, the length of ICU stay, underlying disease, used of NG tube and ventilator were factors associated with NIs rate in internal ICU. In terms of mortality, $62.5 \%$ of the patients in the internal ICU had positive culture and $48.4 \%$ had no infection, and in the ICU surgery $39.7 \%$ of the patients had positive culture and $14.3 \%$ had no infection, which was a significant difference ( $p$-value $<0.001$ ). In a preview studies showed that, NIs rate with increasing of infection (positive-culture) in internal ICU had a significant difference [22-26] Therefore, we should be undertaken to monitor and reduce the affecting factors of the patients in ICUs, who are known as the group with the high risk of NI.

\section{Conclusion}

In our study, it was demonstrated that, positive culture infections were more in the internal ICU compared to the surgical ICU. Age, underlying diseases, the long stay, used of ventilator and NG tube were factors associated with NIs rate in internal ICU. Therefore, we should pay more attention to the influencing factors of critical patients who admitted in internal ICU. It is recommended to, as much as possible to reduce the length of hospital stay in the ICU. If possible, reducing the time makes the patients to use medical equipment, and replacing it will reduce the possibility of bacterial and fungal colonization and 
the application of appropriate prophylaxis for patients prone to infection can be effective in reducing nosocomial infections.

\section{Declarations}

\section{Ethics approval and consent to participate:}

This study was the result of two postgraduate thesis which were approved by Ethics committee of Birjand University of Medical Sciences. The registration number (IR.BUMS.REC.1394-17 and IR.BUMS.REC.139427).

\section{Consent for publication:}

All authors have read and approved the final version

Availability of data and material:

Not applicable

Competing interests:

The authors declare that they have no competing interests

\section{Funding :}

Not applicable

\section{Authors' contributions:}

Carried out the statistical analysis and edited the final version of the manuscript $\mathrm{AE}$, prepared the proposal and practically carried out the study. FN, prepared the paper, edited the final version of the manuscript and helped to carried out the study, EA, carried out the statistical analysis. MZ designed and supervised the study.

\section{Acknowledgment}

The authors presents their special thanks from all the personnel of internal and surgical ICUs as well as the central laboratory of Valia-e-asr Hospital in Birjand for their great help.

\section{References}


1. Herkel T, Uvizl R, Doubravska L, et al. epidemiology of hospital-acquired pneumonia: Results of a Centarl Europen multicenter prospective, observational study compared with data from the European region. Biomed Pap Med FacUnivPalacky Olomouc Czech Repub. 2016;160:448-55.

2. Akbari M, Nejad Rahim R, Azimpour A, Bernousi I, Ghahremanlu H. A survey of nosocomial infections in intensive care units in an imam reza hospital to provide appropriate preventive guides based on international standards. Stud Med Sci. 2013;23:591-6.

3. Kadkhodaei M, Sharif MR, Saba MA, Mousavi GA. Assessing the nosocomial infections'rate and the antibiotic resistance pattern among the patient hospitalized in Beheshti Hospital during 2013. Int Arch Health Sci. 2018;5:11-5.

4. Nadi E, Nekouii B, Mobin A, Nekouii A, MoghimBeigi A. Frequency of Nosocomial Pneumonia in ICUs of Hospitals of Hamadan University of Medical Sciences. IUMS.2011; 29:153.

5. Ding J-G, Sun Q-F, Li K-C, Zheng M-H, Miao X-H, Ni W, et al. Retrospective analysis of nosocomial infections in the intensive care unit of a tertiary hospital in China during 2003 and 2007. BMC Infect Dis. 2009;9:115.

6. Wang L, Zhou K, Chen W, et al. Epidemiology and risk factors for nosocomial infection in the respiratory intensive care unit of a teaching hospital in China: A prospective surveillance during 2013 and 2015. BMC Infect Dis. 2019;19:145.

7. Mancini A, Verdini D, et al. (2016). "Retrospective analysis of nosocomial infections in an Italian tertiary care hospital." The new microbiologica.2016,39: 197-205.

8. Behnke M, Hansen S, Leistner R, Diaz LA, Gropmann A, Gastmeier P, et al. "Nosocomial infection antibiotic use" DtschArzteb/Int. 2013;110:627-33.

9. Iliyasu G, DaiyabFM,Tiamiyu AB, Abubakar S, Habib ZG, Sarki AM, et al. Nosocomial infections and resistance pattern of common bacterial isolates in an intensive care unit of a tertiary hospital in Nigeria: A 4-year review. J Crit Care. 2016;34:116-20.

10. Gao J, Ying K. Homology Analysis and Nosocomial Infection Control on Outbreak of ICU AcinetobacterbaumanniiPneumonia. "CHEST Journal. 2016;149:A93.

11. Mitharwal SM, Yaddanapudi S, Bhardwaj N, Gautam V, BiswalM,Yaddanapudi L. Intensive care unitacquired infections in a tertiary care hospital: An epidemiologic survey and influence on patient outcomes. AmJInfect Control. 2016;44:e113-7.

12. Ellison J, Southern D, Holton D, Henderson E, Wallace J, Faris P, et al. Hospital ward design and prevention of hospital-acquired infections: A prospective clinical trial. Can J Infect Dis Med Microbiol. 2014;25:265-70.

13. Ott E, Saathoff S, Graf K, Schwab F, Chaberny IF. "The prevalence of nosocomial and community acquired infections in a university hospital." DtschArzteb/Int. 2013, 110: 533-540.

14. Chittick P, Sherertz RJ. Recognition and prevention of nosocomial vascular device and related bloodstream infections in the intensive care unit. CritCareMed. 2010; 38: S363-S72.

15. Guggenbichler JP, Assadian O, Boeswald M, Kramer A. Incidence and clinical implication of nosocomial infections associated with implantable biomaterials-catheters, ventilator-associated 
pneumonia, urinary tract infections. GMS KrankenhhygInterdiszip. 2011; 6: Doc 18.

16. Nicholson L. Healthcare-associated infections: the value of patient isolation. Nurs Stand. 2014;29:35-44.

17. -Luzzati R, Merelli M, ansaldi F, Rosin C, azzini A, Cavinato S, et al. Nosocomial candidemia in patients admitted to medicine wards compared to other wards: a multicentre study. Infection. 2016;44:747-55.

18. Zerganipour F, AjamiS,Ketabi S, Samimi A. Critical Success Factors to Control Nosocomial Infection by “Wireless Sensor Network” in Intensive Care Unit. J Inform Tech SoftwEng. 2016;6:1-5.

19. Saleem M, Vaish A, Singh M, Sonkar A, Idrees M, Agarwal J, et al. Nosocomial Infections in the Patients Admitted in the Medicine Ward of a Tertiary Care Hospital in North India: an Epidemiological Study. JRAAS. 2012;28:12-8.

20. Sonmezer MC, Ertem G, Erdinc FS, Kaya Kilic E, Tulek N, Adiloglu A, et al. "Evaluation of Risk Factors for Antibiotic Resistance in Patients with Nosocomial Infections Caused by Pseudomonas aeruginosa. Can J Infect Dis Med Microbiol2016; 2016:1321487.

21. Sahu MK, Siddharth B, Siddharth B, Choudhury A, Vishnubhatla, Singh SP, et al. Incidence, microbiological profile of nosocomial infections, and their antibiotic resistance patterns in a high volume Cardiac Surgical Intensive Care Unit. Ann Card Anaesth. 2016;19:281-7.

22. Zingg W, Holmes A, Dettenkofer M, Goetting T, Secci F, Clack L, et al. Hospital organisation, management, and structure for prevention of health-care-associated infection: a systematic review and expert consensus. Lancet Infect Dis. 2015;15:212-24.

23. Vandijck DM, Labeau SO, Vogelaers DP, Blot SI. Prevention of nosocomial infections in intensive care patients. NursCrit care. 2010;15:251-6.

24. Resende MM, Monteiro SG, Callegari B, Figueiredo PM, Monteiro CR, Monteiro-Neto V, et al. Epidemiology and outcomes of ventilator-associated pneumonia in northern Brazil: an analytical descriptive prospective cohort study. BMC Infect Dis. 2013;13:119.

25. Custovic A, Smajlovic J, Hadzic S, Ahmetagic S, Tihic N, Hadzagic H, et al. Epidemiological surveillance of bacterial nosocomial infections in the surgical intensive care unit. MaterSociomed. 2014;26:7-11.

26. Moreira MR, Guimarães MP, Rodrigues AAdA, GontijoFilho PP. Antimicrobial use, incidence, etiology and resistance patterns in bacteria causing ventilator-associated pneumonia in a clinical-surgical intensive care unit. Rev SocBrasMed Trop. 2013;46:39-44.

\section{Tables}

Table1. Demographic and information of the patients admitted at medical-surgical ICU (MS-ICU) and internal ICU 


\begin{tabular}{|c|c|c|c|c|c|c|c|}
\hline \multirow[t]{3}{*}{ characteristics } & \multirow{2}{*}{\multicolumn{2}{|c|}{$\begin{array}{l}\text { MS-ICU } \\
\text { N=665 }\end{array}$}} & \multirow{2}{*}{\multicolumn{2}{|c|}{$\begin{array}{l}\text { Internal ICU } \\
\mathrm{N}=353\end{array}$}} & \multirow{2}{*}{\multicolumn{2}{|c|}{$\begin{array}{l}\text { Total } \\
\mathrm{N}=1018\end{array}$}} & \multirow[t]{3}{*}{ p-value } \\
\hline & & & & & & & \\
\hline & $\mathbf{N}$ & $\%$ & $\mathbf{N}$ & $\%$ & $\mathbf{N}$ & $\%$ & \\
\hline \multicolumn{8}{|l|}{ Age } \\
\hline$<=20$ & 135 & $(20.3)$ & 21 & (5.9) & 156 & (15.32) & \\
\hline $21-30$ & 93 & $(14.0)$ & 53 & $(15.0)$ & 146 & (14.34) & \\
\hline $31-40$ & 79 & $(12.0)$ & 60 & $(17.0)$ & 139 & (13.65) & \\
\hline $41-50$ & 48 & $(7.2)$ & 25 & $(7.1)$ & 73 & $(7.1)$ & $>0.001$ \\
\hline $51-60$ & 72 & (10.8) & 36 & $(10.2)$ & 108 & $(10.6)$ & \\
\hline $61-70$ & 82 & (12.3) & 53 & (15.0) & 135 & (12.23) & \\
\hline $71-80$ & 92 & $(13.8)$ & 41 & $(11.6)$ & 133 & $(13.06)$ & \\
\hline$>=80$ & 64 & $(9.6)$ & 64 & (18.1) & 128 & $(12.60)$ & \\
\hline \multicolumn{8}{|l|}{ Gender } \\
\hline Female & 229 & $(34.4)$ & 196 & $(55.5)$ & 425 & $(41.74)$ & $>0.001$ \\
\hline Male & 436 & $(65.6)$ & 157 & $(44.5)$ & 593 & $(58.25)$ & \\
\hline \multicolumn{8}{|c|}{ Long term of stay in ICU(day) } \\
\hline \multicolumn{8}{|l|}{$1-2$} \\
\hline $3-4$ & 221 & $(33.2)$ & 68 & $(19.3)$ & 289 & $(28.38)$ & $>0.001$ \\
\hline $5-6$ & 160 & $(24.1)$ & 109 & $(30.9)$ & 269 & $(26.42)$ & \\
\hline 7-10 & 131 & $(19.7)$ & 62 & $(17.6)$ & 193 & $(18.95)$ & \\
\hline$>10$ & 81 & $(12.2)$ & 48 & $(13.6)$ & 129 & $(12.62)$ & \\
\hline Underlining disease & 72 & $(10.8)$ & 66 & $(18.7)$ & 138 & $(13.60)$ & \\
\hline Diabete & & & & & & & $>0.001$ \\
\hline Cancer & 91 & $(13.7)$ & 59 & $(16.7)$ & 150 & $(14.73)$ & $>0.001$ \\
\hline Heart disease & 113 & $(17.0)$ & 17 & $(4.8)$ & 130 & $(12.81)$ & $>0.001$ \\
\hline Chronic lung disease & 155 & $(23.3)$ & 58 & $(16.4)$ & 213 & $(21.0)$ & $>0.001$ \\
\hline Kidnay disease & 140 & $(21.1)$ & 78 & $(22.1)$ & 218 & $(21.42)$ & $>0.001$ \\
\hline Hyper tension & 45 & (6.8) & 20 & (5.7) & 65 & $(6.36)$ & $>0.001$ \\
\hline Risk factors & 206 & $(31.0)$ & 145 & $(41.1)$ & 351 & (34.47) & \\
\hline Urinary catheter & & & & & & & \\
\hline
\end{tabular}




$\begin{array}{|llllllll|}\text { Peripheral catheter } & 665 & 100 & 353 & 100 & 1018 & (100) & \\ \text { Tracheal tube } & 665 & 100 & 353 & 100 & 1018 & (100) & \\ \text { Chest tube } & 262 & 39.6 & 177 & 50.1 & 439 & (43.12) & \\ \text { NG tube } & 72 & 11.1 & 3 & 0.8 & 75 & (7.36) & >0.001 \\ \text { Transfusion } & 438 & 65.6 & 218 & 61.8 & 656 & (64.44) & \\ \text { Central catheter } & 172 & 25.9 & 28 & 7.9 & 200 & (19.64) & \\ \text { Recovery } & 66 & 9.9 & 9 & 2.5 & 75 & (7.36) & \\ \text { Mortality } & 552 & 83.0 & 183 & 51.87 & 735 & (72.2) & >0.001 \\ & 113 & 17.0 & 170 & 48.2 & 283 & (27.8) & \end{array}$

Table 2: Information of the positive culture patients of MS-ICU and internal ICU 


\begin{tabular}{|c|c|c|c|}
\hline \multirow[t]{2}{*}{ characteristics } & $\begin{array}{l}\text { MS-ICU } \\
N=67\end{array}$ & $\begin{array}{l}\text { Internal ICU } \\
\mathrm{N}=96\end{array}$ & p-value \\
\hline & $N(\%)$ & $N(\%)$ & \\
\hline Age & & & 0.01 \\
\hline$<10$ & $3(4.7)$ & 0 & \\
\hline $11-20$ & $3(4.7)$ & $3(14.3)$ & \\
\hline $21-30$ & 16(17.2) & 10(18.9) & \\
\hline $31-40$ & $9(13.2)$ & $11(18.3)$ & \\
\hline $41-50$ & $9(13.2)$ & $4(16.0)$ & \\
\hline $51-60$ & 10(13.9) & 11(18.3) & \\
\hline $61-70$ & $6(7.3)$ & $15(28.3)$ & \\
\hline $71-80$ & $8(11.7)$ & $19(46.3)$ & \\
\hline$>=80$ & $4(6.2)$ & $23(35.9)$ & \\
\hline \multicolumn{4}{|l|}{ Gender } \\
\hline Female & $20(8.6)$ & $47(24.0)$ & 0.13 \\
\hline Male & $48(11)$ & $49(31.2)$ & \\
\hline \multicolumn{4}{|c|}{ Long term of stay in ICU(day) } \\
\hline \multicolumn{4}{|l|}{1} \\
\hline 2 & 0 & 0 & \\
\hline $3-5$ & $1(0.6)$ & $7(10.3)$ & \\
\hline $5-10$ & $3(2.3)$ & $10(9.2)$ & $<0.001$ \\
\hline$>10$ & 16(19.8) & $32(33.3)$ & \\
\hline Diabete & $48(65.8)$ & $47(71.2)$ & \\
\hline Cancer & $5(5.5)$ & $26(44.1)$ & 0.001 \\
\hline Heart disease & $7(6.1)$ & $1(5.9)$ & 0.04 \\
\hline Chronic lung disease & $5(3.2)$ & $21(36.2)$ & 0.09 \\
\hline Kidnay disease & 0 & $24(30.8)$ & 0.42 \\
\hline \multirow[t]{2}{*}{ Hyper tension } & $2(4.4)$ & $10(50.0)$ & 0.02 \\
\hline & $10(4.8)$ & $58(40.0)$ & $<0.001$ \\
\hline
\end{tabular}


Table 3: logestic regression analysis between various factors and medical surgery intensive care unit mortality rate

\begin{tabular}{|lllllll|}
\hline & \multicolumn{3}{l}{ Univariate analysis } & \multicolumn{3}{l|}{ Multivariate analysis } \\
\hline Independent factors & OR & $95 \% \mathrm{Cl}$ & P-value & OR & $95 \% \mathrm{Cl}$ & P-value \\
\hline Positive culture & 0.40 & $0.16-0.96$ & 0.040 & 0.40 & $0.16-0.96$ & 0.040 \\
Age & 1.01 & $1.00-1.02$ & 0.283 & 0.99 & $0.98-1.01$ & 0.283 \\
Gender & 1.05 & $0.67-1.66$ & 0.820 & 0.82 & $0.67-1.66$ & 0.820 \\
the length of ICU stay & 1.20 & $1.05-1.38$ & 0.006 & 0.83 & $0.73-0.95$ & 0.006 \\
Hypertension & 1.24 & $0.62-2.50$ & 0.540 & 1.24 & $0.62-2.50$ & 0.540 \\
Chronic of heart disease & 1.14 & $0.55-2.34$ & 0.724 & 1.14 & $0.55-2.34$ & 0.724 \\
Chronic of lung disease & 0.56 & $0.28-1.12$ & 0.101 & 0.56 & $0.28-1.12$ & 0.101 \\
Chronic of renal disease & 0.62 & $0.27-1.43$ & 0.256 & 0.62 & $0.28-1.43$ & 0.265 \\
Diabetes & 1.22 & $0.59-2.55$ & 0.589 & 1.22 & $0.59-2.55$ & 0.589 \\
Cancer & 0.68 & $0.39-1.18$ & 0.168 & 0.68 & $0.39-1.18$ & 0.168 \\
Blood Transfusion & 0.71 & $0.43-1.17$ & 0.182 & 0.71 & $0.43-1.17$ & 0.182 \\
Chesttube & 1.03 & $0.98-1.08$ & 0.238 & 0.97 & $0.93-1.02$ & 0.238 \\
NGtube & 0.86 & $0.74-1.00$ & 0.049 & 1.17 & $1.00-1.36$ & 0.049 \\
Tracheal tube & 0.92 & $0.84-1.00$ & 0.058 & 1.09 & $1.00-1.20$ & 0.058 \\
Central catheter & 0.81 & $0.41-1.59$ & 0.533 & 0.81 & $0.41-1.59$ & 0.533 \\
\hline
\end{tabular}

OR: odds ratio; $95 \% \mathrm{Cl}: 95 \%$ confidence interval

Table 4: logestic regression analysis between various factors and internal intensive care unit mortality 


\begin{tabular}{|lllllll|}
\hline & \multicolumn{3}{l}{ Univariate analysis } & \multicolumn{3}{l|}{ Multivariate analysis } \\
\hline Independent factors & OR & $95 \% \mathrm{Cl}$ & P-value & OR & $95 \% \mathrm{Cl}$ & P-value \\
\hline Positive culture & 1.12 & $0.49-2.54$ & 0.787 & 1.12 & $0.49-2.54$ & 0.787 \\
Age & 0.96 & $0.95-0.98$ & 0.000 & 1.04 & $1.02-1.06$ & 0.000 \\
Gender & 0.90 & $0.47-1.73$ & 0.760 & 0.90 & $0.47-0.173$ & 0.760 \\
the length of ICU stay & 1.67 & $1.38-2.02$ & 0.000 & 0.60 & $0.49-0.73$ & 0.000 \\
Hypertension & 0.60 & $0.30-1.21$ & 0.151 & 0.60 & $0.30-1.21$ & 0.151 \\
Chronic of heart disease & 0.87 & $0.40-1.90$ & 0.723 & 0.87 & $0.40-1.90$ & 0.723 \\
Chronic of lung disease & 0.34 & $0.16-0.72$ & 0.005 & 0.34 & $0.16-0.72$ & 0.005 \\
Chronic of renal disease & 0.29 & $0.07-1.27$ & 0.101 & 0.29 & $0.07-1.27$ & 0.101 \\
Diabetes & 0.52 & $0.22-1.23$ & 0.137 & 0.52 & $0.22-1.23$ & 0.137 \\
Cancer & 0.04 & $0.00-0.33$ & 0.003 & 0.04 & $0.00-0.33$ & 0.003 \\
Blood Transfusion & 0.78 & $0.21-2.83$ & 0.699 & 0.78 & $0.21-2.83$ & 0.699 \\
Chest tube & 0.00 & - & 0.999 & - & - & - \\
NG tube & 0.68 & $0.57-0.81$ & 0.000 & 1.47 & $1.12-1.76$ & 0.000 \\
Tracheal tube & 0.84 & $0.76-0.92$ & 0.000 & 1.19 & $1.09-1.31$ & 0.000 \\
Central catheter & 1.03 & $0.13-8.04$ & 0.975 & 1.03 & $0.13-8.04$ & 0.975 \\
\hline
\end{tabular}

\section{Figures}

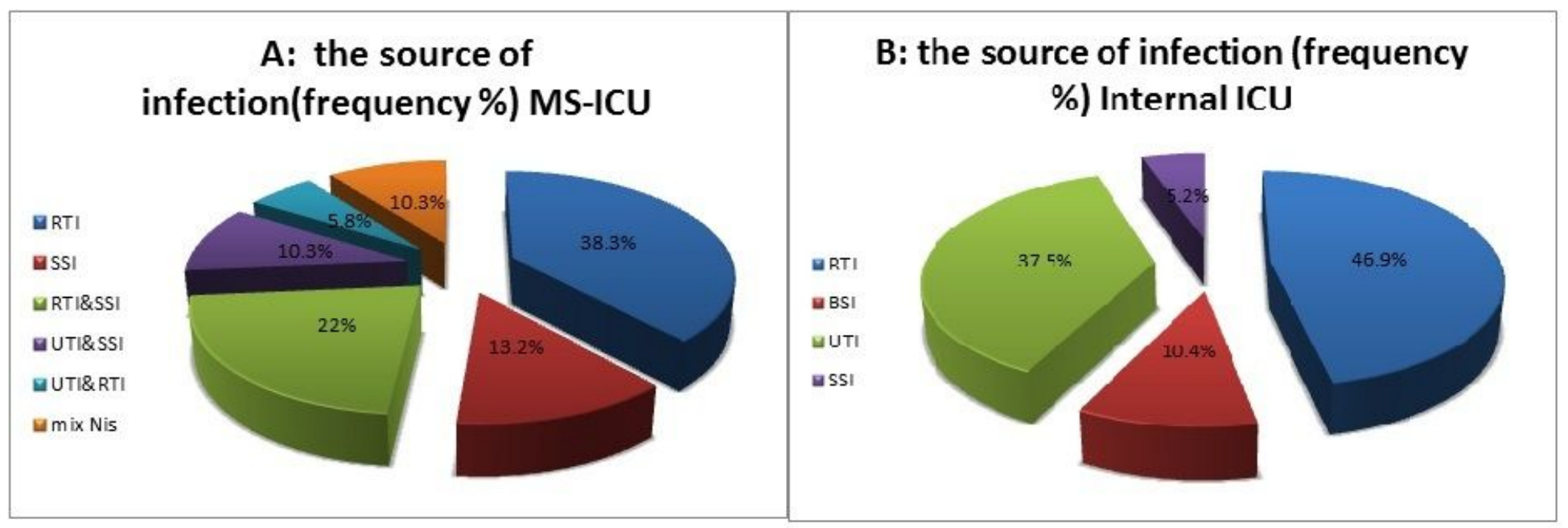

Figure 1 
Depicts the source of infection in number of cases with percentage in internal ICU and MS-ICU.

Respiratory tract infections (RTI); Urinary Tract Infections (UTI); Blood Stream Infections (BSI); Surgery Site Infection (SSI)

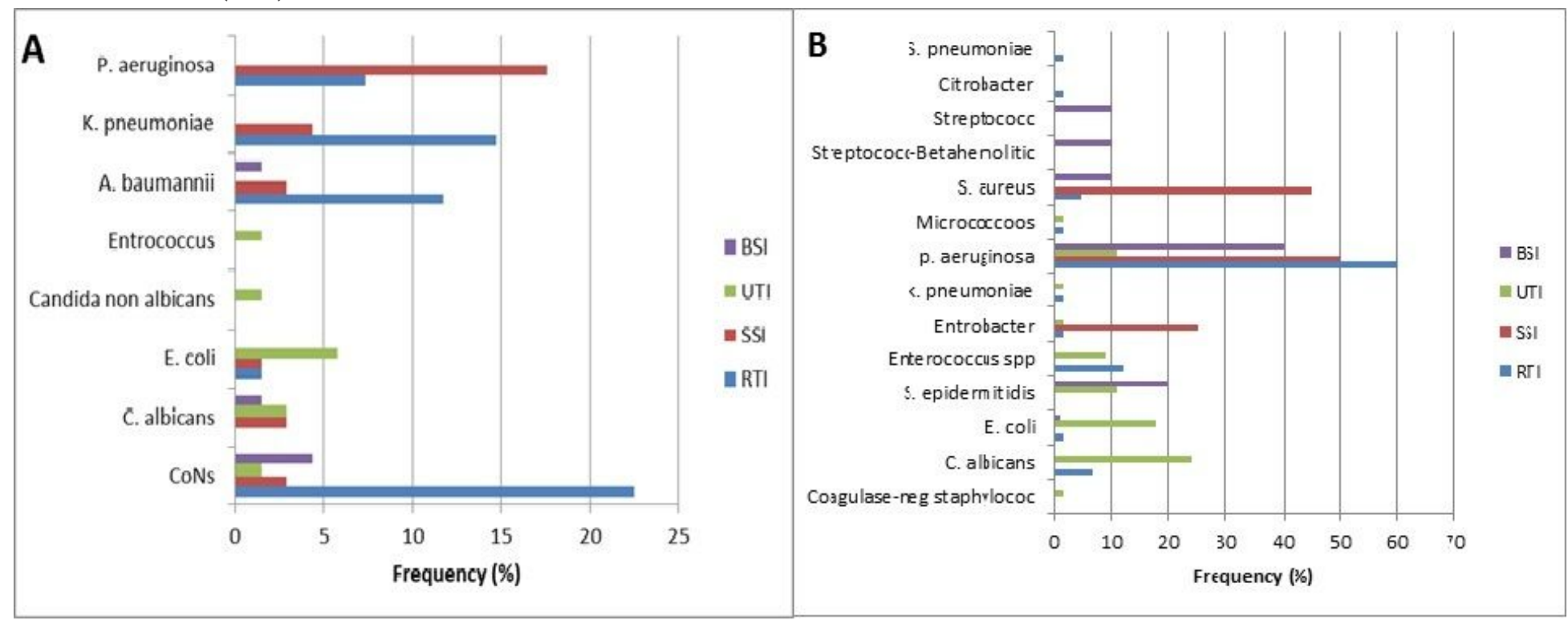

Figure 2

Distribution of causative microorganisms isolated by sites of infections; A: MS-ICU, B: InternalICU. Respiratory tract infections (RTI); Urinary Tract Infections (UTI); Blood Stream Infections (BSI); Surgery Site Infection (SSI). 